fra questa lega e il piombo. Tuttavia, tenendo conto degli errori d'osservazione e dei mutamenti che possono essere arvenuti nella lega e nel piombo nel formarne le asticelle, mi sembra che l'accordo sia sufficiente.

Ho sperimentato poi sulla coppia piombo-lega $\left(\mathrm{Sv}_{18} \mathrm{Cd}_{1}\right)$. I risultati sono nella seguente tabella.

\begin{tabular}{c|c|c}
\hline $\mathrm{T}$ & $\Delta_{1}$ & $\Delta_{2}$ \\
\hline 30 & +94 & -96 \\
17,5 & $\pm 44,5$ & -38 \\
25,0 & +23 & -25 \\
29,4 & +8 & -7 \\
30,6 & \pm 4 & -4 \\
33,0 & \pm 6 & +5 \\
51,2 & $-52,5$ & +60
\end{tabular}

Ia curra che risulta dalla costruzione grafica, taglia l' asse delle temperature a $31^{\circ}, 5$; mentre che nello studio delle correnti termoelettriche fra questa lega e il piombo il punto neutrale fu trovato a $26^{\circ}, 0$. L' accordo pud anche in questo caso ritenersi soddisfacente: e le descritto esperienze confermano bene la teoria.

Sento il dovere di ringraziare il prof. Naccari, che mi porse i mezzi per eseguire queste esperienze.

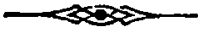

\title{
Comptes rendus.
}

$2^{\circ}$ Semestre 1887.

1. - M. J. Boussinesq. Sulla teoria dell' eflusso per lo stramazzo da sottile parete, quando non vi ha contrazione laterale, $\boldsymbol{e}$ quando la nappa liquida è libera inferiormente. - $\mathbf{E}$ un lavoro analitico pel quale non può farsi un sunto.

M. DI T'oNQUtṙres. Sui movimenti oscillatori e simultanei di due pendoli l' uno sospeso all' altro. - $\mathrm{E}$ ancor questo un interessante lavoro analitico, ma non possiamo che indicarlo.

2. - M. E. MerCadier. Sopra un semplice metodo dinamico per determinare il grado d' isotropia di un corpo solido elastico. - 
68

Lamé indicò con $\lambda \odot \mu$ due quantità caratteristiche di corpo solido, con le quali si esprimono tutti i coefficienti relativi alla elasticitia; $\theta$ secondo il Saint-Venant, in ogni solido veramente isotropo $\lambda$ è uguale a $\mu$. per la qual cosa si puó, misurando quel rapporto, sapere il grado d' isotropia. Ora l'A. dalla sua teoria delle vibrazioni circolari ricava un modo per quella misura.

M. G. CABANelias. Sull' uso del shunt nel metodo balistico. $\dot{\mathrm{E}}$ un lavoro sperimentale per distinguere gli errori possibili impiegando il shunt per un galvanometro. Il principal resultato fu che è sempre cosa giusta di impiegare como shunt in un galvanometro, un numero qualunque di altri galvanometri fra loro identici per ogni riguardo.

M. J. Viol. F. Polarizzazione per emissione. - L'A. ha misurata la proporzione di luce polarizzata $p_{c}$ contenuta in un fascio luminoso emesso dall' argento liquido, impiegando il fotopolarimetro del Cornu, e sotto diverse inclinazioni. I resultati numerici sono quasi matematicamento espressi dalla formula

$$
p_{\mathrm{e}}=(1-\cos i)(1+\cos [75+i / 5]) \text {. }
$$

Siano $e$ ed $r$ i poteri emissivo e riflettente della superficie, - $p_{i}$ la proporzione di luce polarizzata per riflessione; la neutralità del fascio omergente in un ambiente chiuso, secondo una direzione qualunque, esige che $r p_{\mathrm{r}}=e p_{0}$, ed essendo $e=1-r_{2}$

$$
r=\frac{p_{\mathrm{e}}}{p_{\mathrm{e}}+p_{\mathrm{r}}} \text {. }
$$

Non ci è nota $p_{r}$, ma dalle misure esatte del Quincke si può derterminare la proporzione di luce polarizzata nel fascio riflesso alla superficie dell' argento solido. Cosi prendendo per $p_{\mathrm{r}} \mathrm{i}$ valori del Quincke, e per $p_{e}$ quelli dell'A., si calcola $r$ por diverse incidenze.

3. - M. J. Vrolus. Confronto fra le energie irraggiate dal platino e dall' argento fusi. - Uno dei metalli era introdotto nella piccola lampada al decimo del Siemens, la quale consiste in una cassetta con un foro di $0,1 \mathrm{~cm}$. q. dietro al foro $\dot{e}$ il metallo in nastro attraversato dalla corrente, graduata fino a fondere il nastro. I raggi dal foro vanno alla pila termoelettrica, la quale è equilibrata dall' irraggiamento di un' altra sorgente variabile con una nota legge. Si trovò che l'irraggiamento del filo di platino fuso era 54 volte quello dell' argento fuso. Ma questo rapporto è molto minore di quello fra lo intensità luminose.

E. H. Amagat. Solidificazione dei liquidi per la pressione. Secondo la formula di J. Thomson la solidificazione è possibile, so la densità è maggiore allo stato solido che allo stato liquido; 0 questo fu verificato da varii esperimentatori e recentemente dal 
Battelli, ma ancora non si ebbe esempio di solidificazione in virtu della sola pressione. L'A. ha compresso il bicloruro di carbonio in un cilindro di bronzo, superiormente chiuso da un tappo di ferro dolce il quale è il prolungamento del polo di un' elettrocalamita. Vi ha nel liquido un piccolo cilindro di ferro dolce, che attirato chiudendo il circuito batte su quel polo, e col rumore avvisa se il corpo é sempre liquido; a 1500 atm. quel rumore non si udiva più. In altra esperienza il cloruro fu rinchiuso in un pezzo di acciaio che portava due traguardi orizzontali, l'un di fronte all'altro, fatti con coni di vetro rincotto. Un raggio di luce elettrica attraversava questi coni e il liquido fra loro, e cadeva poi in un cannocchiale. Cosi l'A. osservó la solidificazione, non solo, ma anche la formazione di vari gruppi di cristalli nell' interno del liquido, i quali si sono potuti fotografare.

Il cloruro di carbonio si solidifica a

\begin{tabular}{|c|c|}
\hline$-19^{0}, 5$ & alla pressione di \\
\hline 0,0 & \\
\hline 10,0 & \\
\hline 19,5 & \\
\hline
\end{tabular}

La Nota contiene una interessante descrizione del fenomeno osservato al cannocchiale, quando gradatamente si varia la pressione o comincia la solidificazione o il ritorno allo stato liquido.

MI. A. Rigur. Sulla conducibilità del bismuto in un campo magnetico. L' A. enuncia il resultato generale delle sue ricercho su quella conducibilità, e avverte che tal resultato fu pubblicato in sunto nei Resoconti dell' Acc. dei Lincei fino dal 12 giugno 1987, otto giorni prima della Nota pubblicata nei Compt. rend. sullo stesso soggetto. L' A. inoltre, dice in che il suo modo di esperimentare sostanzialmente differisce da qnello del Leduc, ed annunzia che in breve egli pubblicherà per intiero il suo lavoro. N'on diciamo altro di questa Nota perchè potremo aver quel lavoro nel nostro giornale.

4. - M. E. MERCaDiER. Sulla delerminazione del coefficiente di elasticità dell' acciaio. - E una continuazione del lavoro più sopra indicato dello stesso Autore, ma non è suscettibile di un sunto.

MM. P. Ledeboer et G. Maseuvrier. Sul coefficiente di selfinduzione di due rocchetti riuniti in quantita. - Questo lavoro ebbe lo scopo di sapere, se ad un sistema di due rocchetti o spirali, riuniti in quantità, e respettivamente $R_{1}$ ed $R_{2}$ e di coeficienti $L_{1}$ ed $L_{2}$ di self-induzione, si puó sostituire una sola spirale di data resistenza $R$ e di dato coefficiente $L$. Gli Autori trovano che questo non $\dot{e}$ generalmente possibile, eccetto il caso in cui si abbia $L_{1}: R_{1}=L_{2}: R_{2}$ : e allora si ha 
70

$$
\mathrm{L}=\frac{\mathrm{L}_{1} \cdot \mathrm{I}_{2}}{\mathrm{~L}_{1}+\mathrm{L}_{2}}, \quad \mathrm{R}=\frac{\mathrm{R}_{1} \cdot \mathrm{R}_{2}}{\mathrm{R}_{1}+\mathrm{R}_{2}}
$$

Ma vi è un caso ove la soluzione del problema è possibile, indipendente da quell' ultima condizione; ed è quando si determina il coefficiente del sistema delle due spirali col metodo dell'extracorrente, e col ponte di Wheatstono; e gli Autori trovano cho in quest' ultimo caso si ha, indipendentemente dall' anzidetta condizione,

$$
L=\frac{R_{2}^{9} L_{1}+R^{9} L_{1}}{\left(R_{1}+R_{z}\right)^{2}}
$$

Fd esperimentalmente trovarono giusto quest' ultimo valore.

M. E. Doumer. Studio del metallo dei suoni, per mezzo delle fiamme manometriche. - Secondo l'Helmholtz il metallo è solo dovuto agl' armonici del suono fondamentale, e secondo il Koenig può altresi provenir dalle differenze di fase fra il fondamentale $\theta$ gli armonici. Il metodo dell' A. determina l' ordine dell' armonico che accompagna il fondamentale, e determina la differenza di fase fra questi due suoni. Il metodo consiste nella fotografia di una famma manometrica sotto l'influenza del suono da analizzarsi.

L'A. espone solo il metodo, e non si pronunzia sul valore dell' una o dell' altra di quelle teorie. In conclusione esso trova che il suo metodo servo esattamente per lo suddetto determinazioni.

M. A. Berget. Misura della conducibilitì calorifica del mercurio, in valore assoluto. - Sono due cilindri retti concentrici, pieni ambidue di mercurio, con le basi orizzontali; la superiore a $100^{\circ}$, $\theta$ la inferiore a $0^{n}$; la colonna cilindrica interna è separata dalla parte anulare, da un vetro molto sottile; e questa ultima parte essendo circondata da sostanze poco conduttrici, si può considerare la centrale come parte del muro indefinito di cui tratta la nota teoria, e le di cui faccie sarebbero a $100^{\circ}$ ed a $0^{\circ}$. Che nel cilindro centrale le superfici isotermiche f'ossero piane, l'A. lo verifico, adoprando due fili di ferro ricoperti di gutta fuorchè alle estremità; ed ognuno dei fili, era unito ad uno dei reofori di un delicato galvanometro, e quando le loro estremita che servivano a chiudero il circuito, orano immerse in una stessa sezione orizzontale del cilindro verticale di mercurio il galvanometro non dava segno alcuno; e seguitando la esperienza non tenendo quollo cstremita in una stessa sozione, si verifico che la legge delle temperature era una funzione lineare.

Per misurare la quantita di calore che passava in un dato tempo attraverso di una sezione, si fece in modo che quel cilindro fosse il prolungamento della provetta di un calorimetro Bunsen. Questa provetta attraversava una lastra di ferro cho si aggiustava 
esattamente alla base dell' istrumento; e un sottile foglio di baudruche incollato sul tubo e sulla lastra impediva al mercurio di entrare nell' anello cilindrico di mercurio che circondava il cilindro interno. Il tutto poi era immerso nel ghiaccio a zero fino alla base comune ai due cilindri. Con la evaporazione del cloruro di metile si congelava l' acqua stata bollita del calorimetro, si faceva il riempimento del mercurio $\theta$ si aggiustava la bacchetta graduata dell' apparecchio, del quale si era gia fatto uno studio volametrico, quando lo stato normale era stabilito si faceva la lettura del calorimetro misurando il tempo con un cronometro. La colonna cilindrica centrale era $0^{n}, 20$ in altezza e $13^{n \text { n'm, }}, 2$ in diametro; e il tubo di vetro concentrico al primo avea la stessa altezza, e $0^{\infty}, 06$ in diametro. Se $\mathrm{K} \dot{e}$ il coefficiente di conducibilità, fra le temperature $\theta$ e $\theta_{1}$ della colonna di mercurio di lunghezza $l$ e di sezione $A, Q$ la quantita di calore, e $t$ il tempo, si ha, come $\dot{\theta}$ noto nel metodo detto del muro, la formula

$$
\mathrm{Q}=\mathrm{KA} A \frac{t}{l}\left(\theta-\theta^{\prime}\right) \text {. }
$$

$\mathbf{F u}$ trovato $\mathrm{K}=0,02015$.

5. - M. E. MERCadier. Sulla determinazione del coefficiente di elasticità dell' acciaio. - $\mathbf{E}$ una continuazione dello esperienzo ove si applica il metodo di ricerca impiegato nelle precedenti misure, già qui più sopra accennate, ma non suscettibile di un brevo sunto.

M. E. Demarçay. Sugli spettri del didimo e del samario. L'A. osservò in addietro $\operatorname{ch} \theta$ nei prodotti del frazionamento del prasèodimo (praséodyme) vi si osservava la stria $\lambda=469$, con egual forza della $\lambda=444$, con intensità diverse, e concluse che la stria $=469$ non apparteneva al praseodimo. Ora egli dichiara che i resultati delle sue ultime esperienze confermarono quella prima osservazione, ma non quell' ultima conclusione. Nelle fiazioni che non danno più traccia della stria $\lambda=444$, caratteristica del prasèodimo, si vede una stretta striscia $\lambda=469,8$, di aspetto simile alla $\lambda=427,5$ del didimo; e dunque questa striscia non appartiene al prasèodimo. D'altra parte nelle porzioni più pure di quest' ultimo, si osserva una striscia nebulosa $\theta$ diffusa, di $\lambda=469$ circa; e si deve dunque concludere che queste due striscie hanno lunghezze d'onda vicine ma distinte, la prima appartenendo ad un corpo incognito. La Nota termina con alcune altre osservazioni sulle strie osservate sulle porzioni del neodimo puro di prasèodimo, $\theta$ non ancora descritte.

6, 7, 8. - MM. G. Maneuveier e P. Ledeboer. Sul coefficiente $d i$ self-induzione $d i$ due rocchetti (bobines) riuniti in quan- 
tită. - La Nota comincia col rammentare la Nota qui più sopra indicata, che ha lo stesso titolo della prima. Lo scopo di questa ultima, è di ricercare fino a qual grado di approssimazione si verificano $i$ resultati ottenuti nella prima. Fu trovato: $1^{0}$ che generalmente non si può rimpiaz\%are quei due rocchetti con un solo, ecetto il caso detto nella prima Nota. $2^{0}$ che quando si manda nel ponte di Whoatstono una corrente continua, od una extra corrente, $\dot{e}$ sensibilmente ginsto anche il secondo resultato di quella prima Nota, ma non quando si manda nel ponte una serie di extra correnti con l'interruttore girante, od una serie di correnti alternative. $3^{0}$ che quei duo rocchetti possono essere sempre rimpiazzati da un solo, quando le costanti loro sono in un rapporto $L: R$ tanto piccolo, che la durata del periodo variabile sia inferiore al periodo di successione delle correnti discontinue o alternative, lanciate nel ponte.

9, 10. - M. J. Bertrand. Nuova formula per la tensione massima del vapor d'acqua. - Se $k$ e $k c^{\prime}$ sono $\mathrm{i}$ calorici specifici di una sostanza qualunque, abbiamo le note equazioni,

$$
\frac{d k}{d v}=\mathrm{AT} \frac{d^{8} p}{d t^{2}}, \quad \frac{d k^{\prime}}{d p}=-\mathrm{A} \mathrm{T} \frac{d^{2} v}{d t^{2}} .
$$

v o $t$ sono le variabili indipendenti nella prima, $\theta p$ e $t$ nella seconda. Se $k$ e $k$ sono funzioni di $t$ soltanto, i primi membri sono nulli, e dunque son nulli $\frac{d^{2} p}{d t^{2}}$ e $\frac{d^{z} v}{d t^{2}} \cdot p$ (funzione di $t$ o $v$ ) e $v$ (funzione di $p$ e $t$ ) sono funzioni lineari di $t$, e dunque risolvendo 1' equazione che lega $p, v$ e $t$, rolativamente a $t$, si deve avere una funzione lineare di tanto rapporto a $p$ che relativamente a $v$, ossia una equazione della forma

$$
\mathrm{T}=x p v+\beta p+\gamma v+\delta,
$$

$\alpha, \beta, \gamma, \delta$ essendo quantiti costanti.

Da questa equazione, e dalla nota

$$
\left(k^{\prime}-k\right) \frac{d t}{d p} \cdot \frac{d t}{d v}=\mathrm{AT},
$$

si ha.

$$
k^{\prime}-k=\frac{\mathrm{AT}}{(\alpha p+\gamma)(x v+\beta)} .
$$

Ma perchì $k^{t}-k$ sia funzione di $T$, convien che sia $k^{\prime}-k$ costante, ed allora per $\mathrm{T}$ avremmo la forma

$$
\mathrm{T}=\alpha(p+\lambda)\left(v+\lambda^{\prime}\right)
$$


oppure converrebbe che fossero $\beta=0, \gamma=0, T=x p v+\delta$, ossia $p v=\mathrm{R}(\mathrm{T}+\mu)$. Ai gas perfetti corrisponde $\mu=0$. Pei vapori non possiamo considerare $k k^{\prime}$ e $k$ come costanti, presso alla saturazione; ma supponiamo dipendano soltanto dalla temperatura. Allora avremo la (1) oppure la

$$
p v=\mathrm{R}(\mathrm{T}+\mu) \text {. }
$$

Per un vapore la relazione fra $p, v$ e $t$ deve, allontanandoci dalla saturazione, condurre ad un rapporto costante fra $p v$ e $\mathrm{T}$, perchè lo stato limite $\dot{e}$ quello di un gas perfetto. Ora la (1) non soddisfa a questa condizione, ma vi soddisfa la (2), equivalente alla

$$
\frac{p v}{\mathrm{R}}-\mathrm{T}=\mu \text {. }
$$

Cercando le verificazioni numeriche di questa formula per il vapor d'acqua, si trova sensibilmente, costante il rapporto $p v$ : (T +127 ) ed uguale a 2,4760 .

Accettiamo dunque come un fatto la seguente

$$
\frac{p v}{\mathrm{~T}+127}=2,47 \text {. }
$$

Si ha per il calore $r$ di evaporazione, rappresentato esattamente da

$$
r=800-0,705 . \mathrm{T},
$$

la formula

$$
r=\mathrm{AT} \frac{d p}{d t}(\sigma-s)
$$

Trascurando $s$ (volume di un chilogrammo di liquido ed eguale a 0,001 ) ed osservando che $\sigma$ (volume di un chilogrammo di vapore) rappresenta $v$, le (3), (4), (5) conducono a

$$
\frac{d p}{d t} \cdot \frac{1}{p}=\frac{800-0,705 \cdot \mathrm{T}}{\mathrm{AT}(\mathrm{T}+127) 2,47},
$$

Nella (5) la unità di pressione, ò la pressione di un chilogrammo sopra un metro quadrato, e nella (6) $\dot{e}$ la pressione di un millimetro di mercurio, $\theta$ bisogna dunque moltiplicare il secondo membro della (6) per 760: 10333; e calcoli fatti abbiamo

o quindi

$$
\frac{d p}{d t} \cdot \frac{1}{p}=\frac{79,714}{\mathrm{~T}}-\frac{88,635}{\mathrm{~T}+127},
$$

$$
p=\mathrm{G} \frac{\mathrm{T}^{\mathrm{a}}}{(\mathrm{T}+127)^{\mathrm{b}}}, a=79,714, \quad b=88,635 .
$$


74

G si determina con un caso particolare; e p. es. facendo $\mathrm{T}=\mathbf{3 7 3}$ dovendosi avere $p=760$. Assumendo

$$
\log G=34,21083,
$$

si trova la (8) rimarchevolmente d' accordo con le tavole del Regnault, massime ponendo 126,37 invece di 127 , ed $a=79,628$, $b=88,578$.

11, 12. - M. J. Bertrand. - M. Bertrand fa omaggio all'Accademia delle sue Isezioni sulla termodinamica, e presenta alcune osservazioni relative alla cosi detta funzione di Carnot. In queste osservazioni egli si domanda il perchè quella funzione fu scoperta tanto tardi, e poi arriva a concludere che ciò accadde perchè Clapeyron, il sapiente commentatore del Carnot, non considerò che dei cicli infinitamente piccoli; ed in questo caso la loro superficie $\dot{\theta}$ un infinitamente piccolo di secondo ordine, ed è dunque trascurabile. Or siccome $\dot{e}$ essa che fa tutta la differenza fra la teoria del Mayer e quella del Carnot, le due teorio per essa coincidono.

L' A. ha determinata la forma che Carnot e Clapeyron avrebbero dovuto dare a quella funzione secondo i loro principi; forma molto diversa da quella che i progressi della scienza hanno fatto accettare.

13. - M. E. Gossart. Ricerche sullo stato sferoidale. - I'A. si è proposto di determinare col calcolo $\theta$ con la esperienza, la semi-sezione meridiana di una goccia liquida, allo stato di calefazione sopra una lastra orizzontale. A tale oggetto esso ricorre alle formule della teoria della capillarità, fa uso della fotografia istantanea ed illumina la goccia con le scintille di un rocchetto di Rubmkorff; ma in questa Nota non descrive che il suo calcolo.

14. - M. Mascart. Alcune proprietà relative all' azione delle lamine cristalline sulla luce. - Un sunto di questa Nota non sarebbe possibile, e converrebbe tradurla per intiero; ma, non potendo far questo, ci limitiamo all' enunciato del seguente teorema dimostrato nella Nota.

L' azione sulla luce di un insieme qualunque di lamine cristalline, possedendo o no un potere rotatorio, equivale a quella di una sola lamina di un cristallo ad un asse, parallela all'asse $\theta$ normale ai raggi incidenti. Questo stesso teorema puó enunciarsi più generalmente come segue.

Qucsta medesima axione di un insieme di lamine cristalline, equivale a quella di una sola lamina di un cristallo ad un asse, parallela all' asse, e normale ai raggi incidenti, i quali indebolirebbero nella stessa proporzione $\mathrm{i}$ raggi ordinario e strordinario. 
M. Sfмmola. Sul riscaldamento delle punte nella scarica elet. trica. - Si ha una coppia antimonio e bismuto che termina in punta, fa parte del circuito di un galvanometro, e comunica col conduttore di una macchina elettrica. Quando questa agisce si ha deviazione nel galvanometro. Si ha lo stesso fatto, facendo che la coppia comunichi col suolo, e tenendola presso quel conduttore. Nell' oscurita, si vede che quando sulla punta apparisce il punto luminoso, o stelletta, la deviazione del galvanometro è la massima; - ciò, dice l'A., prova che la scarica negativa sviluppa più calore della negativa. Quando la punta è cosi vicina al conduttore che la scintilla è continua, quella deviazione ò molto minore. Il cosi detto venticello elettrico è caldo per so stesso; e ciò si prova fissando sul conduttore una punta metallica ricurva, ed a poca distanza dalla faccia di una pila Nobili. L'A. propone di sustituire quella punta termoelettrica, alla punta ordinaria di un parafulmine, per esplorare la elettricità atmosferica.

MM. P. Ledeboer e G. Maneuvrier. Sull' uso e sulla graduazione dell' elettrometro a quadranti, nel metodo omostatico. Per quel metodo omostatico l'A. indica il lavoro di M. Joubert Studi sulle macchine elettromagnetiche (Ann. de l'École Normale). Gli Autori avevano l' elettrometro a settori del Curie, ove i settori sono di acciaio, magnetizzati, e l' ago di aluminio è sospeso ad un filo sottilissimo di platino, col diametro di un quarantesimo di millimetro. Adoperando questo apparecchio hanno veduto che le deviazicni dell' ago non erano le stesse, in parità delle note circostanze, da un lato e dall' altro della posizione normale, ed hanno scoperto che tal cosa proveniva da una differenza di potenziale che normalmente preesisteva, fra l'ago di aluminio ed i settori di acciaio; differenza che essi hanno misurata pel loro apparecchio.

$\mathrm{X}$.

H. EBERT. Sulla relazione fra la lunghezza d' onda e l' intensità luminosa. - Wiedemann's Annalen, 1887, n. 11. - Uno fra i problemi più importanti dell' ottica è quello di sapere se la velocità $V$ di propagazione della luce (e quindi, anche la lunghezza d'onda) varia coll'intensità. Se una tal variazione esistesse, mentre ci sarebbe utile in alcuni problemi d'astronomia fisica (p. es. dalla variazione di lunghezza d' onda osservata dedurre il movimento relativo dolla sorgento luminosa), indicherebbe contrariamente alle teorie attuali l' esistenza di un attrito nell' etere.

J. J. Muller ${ }^{1}$ ) con un metodo, d' altronde soggetto a grandi errori d'osscrvazione, aveva trovato che il valore di $\mathrm{V}$ aumenta

1) Pogg. Ann. t.: 145, pag. 86, 1871. 
coll' intensita da 1,5 a 5 chilom. secondo il colore; mentre secondo F. Lippich ') V sarebbe invariabile. Ebert ha ripreso percio la questione, adoprando il metodo delle interferenze nelle lamine di vetro spesso a faccie quasi parallelo.

Se due raggi interferiscono con una differenza di cammino di 20000 ondulazioni ed è possibile osservare una variazione in questa differenza di $1 / 10$ della lunghezza $d$ ' onda $\lambda$, si potrà constatare una variazione di $1 / 230000$ nel valore di $\lambda$, corrispondente $a \pm 1,5 \mathrm{~cm}$. in V. Sia $\Delta$ lo spessore della lamina in un punto qualunque; nel caso dell' incidenza normale vi sarà un minimo d'intensita per ogri $\Delta=\frac{2 m \lambda}{4}$, e la distanza in millimetri di due frangie consecutive sarà $\frac{\lambda}{4 \operatorname{cotg} \alpha}, m$ essendo un numero intero qualunque ed $x$ l' angolo delle due superfici. Quest' angolo deve essere molto piccolo per potere apprezzare le frazioni della distanza fra due frangie consecutive: variava da $7^{\prime \prime}$ a $31^{\prime \prime}$ a seconda delle lamine, che avevano uno spessore da $1^{\mathrm{min}}, 25$ a $7 \mathrm{~mm}, 49$; cosicché, le differenze di cammino per i colori medii erano di 6300 a 37500 oscillazioni. Quando $\lambda$ varia di $d \lambda$, il sistema di frangie subisce uno spostamento di $\frac{d \lambda}{4 \operatorname{cotg} \alpha}$. Essendo impossibile misurarlo con un reticolo, le lamine furono tagliate in direzione normale alle frangie d'interferenza $\theta$ riunite rovesciate in modo che spostandone una, si faceva coincidere una delle frangie dei due sistemi. Cosi fu possibile, se non di misurare, di constatare uno spostamento di $1 /$ so $\lambda$.

La sorgente di luce omogenea e costante, come necessita in queste ricerche, fu ottenuta: $1 .^{\circ}$ ) con fiamme colorate da sali di $\mathrm{Na}, \mathrm{Li}, \mathrm{Sr}, \mathrm{Tl} ; 2 .^{\circ}$ ) coi sali $\mathrm{Sr}$ e $\mathrm{Tl}$ nel folgoratore di Lockyer; $\left.3 .^{\circ}\right)$ con tubi di Geyssler ordinari contenenti $\mathbf{H}$ o vapori di $\mathrm{Hg}$. Per mantenere costante l' intensita della fiamma, furon regolate con un apparecchio assai complicato la pressione e la quantità di gas, o cosi pure la pressione e la quantita d'aria che manteneva la combustione e che prima d'arrivare alla fiamma, mediante un apparecchio simile agli iniettori delle caldaie, polverizzava una soluzione molto diluita del sale, il quale era cosi introdotto sempre nelle stesse condizioni nella fiamma. Le medesime soluzioni servirono nol folgóratore di Lockyer, il di cui apparecchio d'induzione (come anche per i tubi di Geissler) agiva con una corrente costante $\theta$ con interruttore ben regolato.

Un prisma a vision diretta produceva di questa luce uno spettro

1) Wiener Berichte, t. 72, par. 355, 1875. 
verticale, di cui una riga era reflessa dall' ipotenusa d' un prisma rettangolo, sull' apparecchio d'interferenza composto di una lente e di una delle lamine già descritte. Osservando le frange mediane e coincidonti, dall' alto ed a traverso di un diaframma molto stretto che non lasciava passare se non raggi paralleli, si puó constatare senza timore di errori soggettivi, il minimo spostamento della frangia oscura.

Con 12 vetri affumicati, che assorbono quasi egualmente tutti i colori, e variamento combinati si poteva ridurre l'intensità luminosa fino a $1 / 250$ del valore primitivo. Colla fiamma del $\mathrm{Na}$ p. es. la lamina avendo uno spessore di 25000 ondulazioni, era possibile osservare una variazione di $1 / 5000000$; ora invece, qualunque fosse l' intensita luminosa, la posizione delle frange rimase invariabile.

Diamo qui i resultati analoghi per le varie righe osservate:

\begin{tabular}{c|c|c|c}
\hline $\begin{array}{c}\text { Qualità } \\
\text { della luce }\end{array}$ & $\begin{array}{c}\text { L'intens. luminosa } \\
\text { fu ridotta a }\end{array}$ & $\begin{array}{c}\lambda \\
\text { si è trovata }\end{array}$ & $\begin{array}{c}\mathrm{v} \\
\text { costante fino a : }\end{array}$ \\
\hline $\mathrm{Li}_{x}$ & $1 / 10$ & $1 / 300000$ & $1,0 \mathrm{chil}$. \\
$\mathrm{H} x$ & $1 / 34$ & $1 / 340000$ & 0,9 \\
$\mathrm{Nax}$ & $1 / 34$ & $1 / 500000$ & 0,6 \\
$\mathrm{Hg}_{x}$ & $1 / 250$ & $1 / 540000$ & 0,4 \\
$\mathrm{Tl}_{\chi}$ & $1 / 20$ & $1 / 860000$ & 0,3 \\
$\mathrm{H} \beta$ & $1 / 10$ & $1 / 320000$ & 1,0 \\
$\mathrm{Sr}_{\delta}$ & $1 / 9$ & $1 / 340000$ & 0,9 \\
$\mathrm{Hg}_{\beta}$ & $1 / 10$ & $1 / 520000$ & 0,6
\end{tabular}

Conclusione. La lunghezza d'onda (e perciò la velocità di propagazione) della luce è quasi fino a $1 / 1000$ 000 indipendento dalla sua intensità, quando questa varia da 1 a 250 .

Unendo a questo fatto altre osservazioni, come la coincidenza esatta delle righe di Fraunhofer con quelle delle nostre sorgenti luminose, l' Autore crede di poter dire cho il suo teorema $\dot{0}$ vero per qualunque intensità conosciuta.

B. Dessat.

H. EBERT. Sull' influenza dei valori limiti della sensazione di luce sul carattere degli spettri. - Wied. Ann. XXXIII, 1888, p. 136. - Anche nell' oscurita assoluta esiste sempre una seusazione vaga di luce dovuta a processi fisiologici interni. L' Autore ha determinato per $i$ diversi colori l'intensita luminosa minima cho deve avere un processo esterno, per produrre una sensazione percettibile accanto alla sensazione costante interna. La cosa era stata 
fin qui studiata solo da Aubert ') che aveva trovato per tale intensitá minima $1 / 300$ della luce emessa da un foglio bianco esposto alla Iuna piena.

La luco emessa da una parte della fiamma Argand, presa $1 \mathrm{~cm}$. al disopra del bruciatore, produceva sopra un foglio oleato una superficio d'intensita luminosa uniforme, e di qui veniva proiettata sulla fonditura di uno spettroscopio per mezzo d'una lente di $12 \mathrm{~cm}$. d'apertura. Una fenditura oculare mobile limitava l'osservazione ad una piccola parto dello spettro, e un diaframma di cm. 0,07 di diametro spostandosi fra la lente e il collimatore veniva a far variaro la quantita di luce utilizzata. La distanza fra il collimatore e il diaframma esseado $\mathrm{E}$, fra il collinatore e la lente $125 \mathrm{~cm}$., il diametro $\mathrm{D}$ utilizzato della lente, sarà: $\mathrm{D}=\frac{12 \breve{5} \times 0,07}{\mathrm{E}} \mathrm{cm}$, e l' intensità luminosa d' una parte qualunque dello spettro sarà proporzionale a $\mathrm{D}^{2}$. Si sposta il diaframma finchè la sensazione luminosa sparisce : si avvicina finchè appare di nuovo, e le due distanze $\mathbf{E}$ danno i limiti inferiore $\theta$ superiore della sensazione minima, poichè l' occhio può seguire un'impressione evanescente fino ad una intensità molto minore di quella necessaria per rendere percettibile un' impressione nascente. La media geometrica dei due valori dà l'intensita cercata. Gli osservatori furon due $\theta$ i valori trovati sufficientemente concordanti. $L$ ' inversa di questi valori, moltiplicata por un numero costante, misura la sensibilità relativa. L'A. trovò, per la luce del gaz, rosso $1 / 192$, giallo $1 / 4$, verde 1 , verde bluastro $1 / 1,6$, bleu ${ }^{1 / 14}$. Calcolando poi le energie relative dei varii colori di una famma a gas, l' A. giunge a questo teorema: che per l'occhio normale l'energia vibratoria necessaria a produrre una sensazione luminosa è minima, quando la lunghezza d' onda è quella dei raggi verdi. Ciò spiega le osservazioni di Weber e Stenger che spesso un corpo emette dapprima della luce verde, e il fatto delle nebulose poco lucenti il cui spettro contiene sopra tutto dei raggi verdi.

B. Dessat.

H. EBERT. Influenza dello spessore e della intensita dello strato radiante sul carattere dello spettro. - Wied. Ann. XXXIU, p. 15̆6. - Secondo Wüllner ${ }^{2}$ ) gli spettri di righe possono trasformarsi in spettri cannellati, variando la pressione del gas luminoso, o lo spessore osservato. Ebert variando con un vetro cuneiforme l'intensità della luce emessa, o allontanandosi con uno spettroscopio a vision diretta dalla sorgente luminosa, ridusse uno spettro cannellato alle 4 righe caratteristiche, secondo Wüllner,

1) Elementi d' ottica fisiologica, Lipsia 1876.

2) Corso di Fisica. 
dello spettro di righe. Viceversa, concentrando la luce della sorgente luminosa, si puó molto prossimamento passare allo spettro cannellato. Causa della trasformazione dello spettro son dunque dei cangiamenti molecolari.

B. Dessat.

WIEdemann e EBert. Sull' influenza della luce sulle scariche elettriche. - Wied. Ann. XXXIII, p. 241. - Aumentando la distanza delle punte di un rocchetto d'induzione, finchè non passi più la scintilla, essa passa di nuovo quando si dirige sulle punte dei raggi ultra-violetti. L' esperienza è di Hortz ${ }^{1}$ ). Ebert e Wiedemann adoprarono una macchina di Holtz, i di cui poli comunicavano con un micrometro $d a$ scintille, e per derivazione con due sfere di platino racchiuse entro un tubo, dove variava la pressione $\theta$ la natura del gas. Ia luce di una lampada elettrica molto ricca in raggi ultra-violetti, era concentrata da una lente di quarzo sulle due sfere. Quando la scarica passava a stento fra le sfere del micrometro si dirigeva subito sulle due sfere di platino appena venivano illuminate; e bisognava riavvicinare le prime di $1 / 3$ perchè la scarica passasse di nuovo. Inoltre il numero delle scintillo aumentava da 3 a 4, e d'irregolari che erano divenivano d' una regolarità perfetta. Cosi pure, introducendo un telefono nel circuito si aveva un suono puro sotto I' influenza della luce, ed un rumore senza questa. Questi effetti si osservano però illuminando l' elettrodo negativo, e mancano quando esso è protetto dalla luce. Lo stesso fu osservato quando si operò coll' elettricità positiva o negativa soltanto, l'altro polo essendo posto al suolo.

Rarefacendo l'aria, secca od umida che sia, gli effetti aumentano fino ad una certa pressione, diminuiscono in seguito e a 100 millimetri spariscono. Cosi fu osservato nell' $\mathrm{H}$ e nel $\mathrm{CO}_{2}$, pei quali gas videsi ancora cho tutti i raggi compresi fra le righe $\mathbf{G}$ e $\mathbf{K}$ son capaci di produrre il fenomeno in questione, mentre rimangono inattivi i raggi calorifici.

Non è da pensare ad effetti d'assorbimento dei gas che ricoprono la superficie degli elettrodi, poichè vi è analogia completa in gas di un potere assorbente molto diverso. Poichè $i$ fenomeni avvengono colla sola elettricità negativa, secondo $\mathrm{E}$. Wiedemann la durata d'ondulazione dei raggi dei catodi si avvicinerebbe a quella dei raggi ultra-violetti, che per conseguenza favorirebbero la produzione di vibrazioni sincrone.

B. Dessat.

1) Wied. Ann. XXXI, 1987, pag. 988. 
80

Ann. der Phy. und Ch. von G. Wiedemann. XXIX, 1886.

11. (Continuaz.) - S. v. Wroblewskr. Relazioni fra lo stato liquido e lo stato solido della materia, rese evidenti con l' uso delle linee $d^{\prime}$ ugual densità. - $\dot{\mathrm{E}}$ noto che in $\nabla e c \Theta$ delle ordinarie isoterme il Jamin propose, per rappresentare lo stato dei fluidi, di considerare la densità e la pressione anzichò il volume $\theta$ la pressione. L'A. propone un nuovo modo di rappresentare la correlazione fra lo stato liquido e quello aeriforme, studiando la dipendenza fra i valori della temperatura e della pressione per ogni dato valore della densità del corpo. Cioè: se una certa quantiti di gas o di liquido ha una densità determinata a una data pressione $\theta$ a una data temperatura, al cambiare della temperatura dovrà cambiare anche la pressione perchè la densità rimanga la stessa; e sono le curve che rappresentano questa dipendenza fra temperatura e pressione, chiamate isopicne, o di ugual densità, che sono studiate dall' A. per l' anidride carbonica, (per la quale sono più numerosi i dati sperimentali occorrenti). In un diagramma annesso alla memoria son disegnate lo curve per lo densita comprese fra 1,2 e 0,025. Il passaggio da una curva all' altra, tanto ncl senso delle ascisse (temperature assolute), quanto in quello delle ordinate (pressioni) dà un' idea della compressibilità e della dilatazione della sostanza per effetto del calore. Nel diagramma è disegnata anche un'altra curva, chiamata curva di liquefazione, o curva fondamentale, che è l'insieme di due curve: la prima delle quali si ottione rappresentando fino alla temperatura critica la variazione della tensione massima colla temperatura; e l'altra, che si ha per temperature al di sopra del punto critico, si ottiene, rappresentando invece delle tensioni massime, quelle pressioni per le quali per ogni temperatura il prodotto della pressione pel volume è minimo.

Secondo il concetto di Andrews, la sostanza è liquida nelle condizioni rappresentate alla sinistra del diagramma al di sopra della curva di liquefazione fino all' ordinata condotta per la tem. peratura critica, ed è gasosa in, tutto il resto del campo. In vece, secondo l'A., se una divisione fra i due stati della sostanza deve farsi è questa: che ciò̀ essa è gasosa al di sotto della curva di liquefazioie, $\theta$ liquida al di sopra, come resulta dalla considerazione della compressibilità della sostanza, misurata col valore del quoziente $\frac{d_{1}-d}{p_{1}-p}$, ove $d_{1}$ e $d$ sono le densità corrispondenti a due isopicno contigue, $\theta p_{1}-p$ è la differenza di pressione misurata su un' ordinata compresa fra di esse. In fatti quel quoziente subisce 
Io stosse variazioni tanto per le ordinate situatc alla destra, quanto per quelle situate alla sinistra delle ordinate relative alla temperatura critica. Lo stesso vale per i coofficienti di compressibilita $k=\frac{1}{v} \frac{v_{1}-v_{2}}{p_{1}-p_{2}}$, ove. $v \dot{\mathrm{o}}$ il volume del corpo alla pressione di 1 atm. e alla temperatura dell' esperienza, mentre $v_{1}$ e $v_{2}$ sono i volumi che ha alla stessa temperatura e alle pressioni $p_{1}$ e $p_{2}$. Le curvo che riuniscono $i$ punti che hanno ugual coefficicnte di compressibilità non subiscono nessuna discontinuità sull' ordinata relativa alla temperatura critica, e lo stesso vale per la dilataziono termica; talchè l'ordinata condotta per la temperatura critica non puó ritenersi qualo limite fra lo stato liquido e quello gasoso; $\theta$ perció il ritenere che la temperatura critica sia la temperatura al di sopra della quale è impossibile la liquefaziono del gas è un concetto errato, e deve abbandonarsi come nocivo per $i$ futuri progressi della scienza.

In fatti Andrews fondo le suo deduzioni sulla impossibilita di ottenere per la $\mathrm{CO}_{2}$ il menisco di separazione fra liquido e gas al di sopra della tomperatura di $30^{\circ}, 92$; ma Jamin fece osservare che ciò non dimostra che sopra tal temperatura critica un gas, sotto pressione conveniente, non sia riducibile allo stato liquido, ma che la mancanza del menisco dipende soltanto dall' esser piccolissima, alla temporatura critica, la differenza fra la densita del vapor saturo e quella del liquido; differenza che per altro non si annulla mai.

Secondo l'A. non prova nulla nemmeno l'andamento delle isoterme al di sopra e al di sotto della temperatura critica, perchè la flessione dolle isoterme relative a temperaturo inferiori a quella critica è dovuta alla notovole differenza fra le densità del vapore $\theta$ del liquido.

Contro le idee esposte, dice l'A., non si puó opporre che $\dot{\theta}$ impossibile parlare di stato liquido al di sopra della temperatura critica, perchè al di là di essa temperatura non si ha piu calor latente; poichè prima di tutto quosto annullarsi del calor latente alla temperatura critica non $\dot{\theta}$ in accordo colle esperienze; e poi l'A. ritiene che fra le calorie di vaporizzazione di un liquido e quelle necessarie all' espansione di un gas non vi sia nessuna differenza, o che ambedue que' fenomeni sieno soltanto gradi diversi di uno stesso processo, che è il livoro nccessario a vincere le forze mulecolari. Quindi alla temperatura critica il calore di evaporazione non si annulla, ma converge verso il calore di espansione, che $\dot{o}$ assorbito dal gas che ha una densità quasi uguale a quella del liquido e la cui pressione è infinitamente poco diminuita. 
La densità del vapor saturo alla temperatura critica non d̀ uguale a quella del liquido, perchè le isopicne corrispondenti non coincidono fra loro.

Da tutto ciò resulta che il punto critico di Andrews deve ritenersi soltanto como esprimente quella temperatura al di sopra della quale non si può osservare la formazione del menisco; $\theta$ in vece si dovrebbe introdurre il concetto della densitì critica, che sarebbe la più piccola densità che il corpo può avere allo stato liquido. Per l'acido carbonico questa densità è $0, \overline{\mathbf{a}}$ e l' isopicna corrispondente si confonde quasi colla curva fondamentale del diagramma. Lo isopicne corrispondenti a densità più piccole sono al di sotto della curva fondamentale, le altre sono al di sopra.

E. Budne. Su un modo di decidere fra la legge fondamentale elettrodinamica del Weber e quelle del Riemann e del Clausius. Se entro un corpo conduttore $\mathrm{H}$ si sospende un magnete col suo asse verticale, $\theta$ se ad $H$ si comunica una carica $E$ di elettricita, secondo la teoria di Clausius tale carica non deve avere nessuna azione sul magnete, secondo le altre teorie in vece il magnete deve ricevero un impulso che produce uno spostamento angolare misurato da

$$
\begin{aligned}
& 2 / 3 \sqrt{k} \frac{\mathrm{NV}}{\sqrt{\tau}-\mathrm{M}} \text { secondo Weber, } \\
& 2 \sqrt{k} \frac{\mathrm{NV}}{\sqrt{\tau \mathrm{M}}} \text { secondo Riemann, }
\end{aligned}
$$

se $\mathrm{V} \dot{e}$, in misura elettrostatica, l'aumento di potenziale all' interno per la carica $E, \sqrt{k} \dot{o}$ il rapporto fra le unità elettrostatiche e quelle elettromagnetiche, $\mathrm{N}$ il momento del magnete in misura magnetica, $\tau$ è la coppia direttrice del magnete, ed $M$ il suo momento d' inerzia (compresovi lo specchio).

L'A. ha calcolato che adoprando una sfera eava $\mathrm{H}$ caricata per una distanza esplosiva di $2 \mathrm{~cm}$. (facendo $\mathrm{V}=200 \mathrm{C}$. G. S.) o un magnete lungo $15 \mathrm{~cm}$. $\theta$ del diametro di $1 \mathrm{~mm}$., si dovrebbe avere, secondo la formula del Weber, la deviazione di $1 \mathrm{~mm}$ su una scala distante $6 \mathrm{~m}$. dallo specchio, purchè $\sqrt{\tau \mathrm{M}}$ sia circa $1 / 200^{\circ}$ Secondo la formula del Riemann tal deviazione deve essere tripla, e per ampliticarla si potrebbe anche adoprare opportunamente una grossa bottiglia di Leyda ben isolata.

L'A. non ha potuto eseguire tali esperienze, che sarebbe lieto di vedere eseguire da chi ne avesse i mezzi.

J. KoLIER'. Su un nuovo galvanometro. - Il sistema magnetico consiste in due sottili molle da orologio piegate ad arco di cerchio, col raggio del cerchio uguale a $3 \mathrm{~cm}$., lunghe $1 / \%$ della 
circonferonza e riunite fra loro con una leggerissima sospensione in modo che tutto il sistema sia girevole attorno al centro del cerchio del quale i due magneti fanno parte. I poli dei due magneti son disposti in modo che il sistema sia astatico. Le estremità dei magneti sono mobili entro rocchetti cilindrici che sono disposti agli estremi di due diametri del cerchio anzidetto, che fanno fra loro un angolo di circa $72^{\circ}$. Ogni rocchetto ha il raggio interno $\mathrm{di} \mathrm{cm}$. 0,35 quello esterno $\mathrm{di} \mathrm{crn}$. 1,25 e contieno 4000 giri di filo isolato da telefoni dello spessore di $1 / 18$ di $\mathrm{mm}$.

Con questo galvanometro, e con un sistema astatico che avesse la durata di oscillazione di 60;, si dovrebbe avere secondo l' A. una deviazione di 0,7 divisioni della scala (distante 1355 divisioni) per una corrente di 0,000 000 001 ampère; sarebbe ciò̀ assai più sensibile del galvanometro Rosenthal.

12. - C. Fromm.. Sulla polarizzazione galvanica prodotta da piccole forze elettromotrici. - $\mathrm{L}^{\prime} \mathrm{A}$. ha adoperato piccole forze polarizzanti, tali cioè che con esse non si abbia scomposizione visibile dell' acqua, per poter far uso del metodo di Fuchs, metodo che consiste nel porre al di dietro dell' elettrodo di cui si vuole studiare la polarizzazione, e percio fuori del circuito, un terzo elettrodo che resta neutro e che si collega con una coppia di quadranti di un elettrometro, mentre I' elettrodo polarizzato $\dot{e}$ in comunicazione con l'altra coppia di quadranti che è derivata a terra. Cosi è possibile di misurare la polarizzazione di ciascun elettrodo durante l' azione della forza polarizzante, $\operatorname{com} \theta$ pure di studiare la diminuzione della polarizzazione dopo che il circuito primario è stato interrotto.

In questa $\theta$ in altre tre Memorio contenuto nel vol. XXX di questi Annali, l' A. ha.studiato la polarizzazione con elettrodi di $\mathrm{Pt}$, Au o $\mathrm{Pd}$, e per spiegare i resultati ottenuti fa le ipotesi seguenti, che egli dichiara di ritenere non le sole possibili, ma almeno le più semplici. Egli adunque ritiene: $1^{0}$ che con qualunque forza elettromotrice, anche con le più piccole che possono adoprarsi, abbia sempre luogo la separazione dell' $H$ e dell' $O$ dall' acido solforico diluito adoprato come elettrolita: $2^{\circ}$ che ambedue $\mathrm{i}$ gas $\mathrm{H}$ - O possano penetrare entro gli elettrodi. Su quest' ultimo punto l'A. fa notare che è già conosciuta l' occlusione dell' $H$ nel $\mathrm{Pt} O$ nol Pd: le esporicnzo cho egIi ha eseguito farebbero ritenere cho anche l'oro presentasse talo occlusione; e che essa si dovesso avere anche per l'ossigeno.

Per la cognizione dei resultati ottenuti, $\theta$ pel modo con cui 8ono spiegati mediante le due ipotesi accennate, vedansi le 4 estese Memorie dell' A., delle quali non è possibile dare un breve sunto. 
F. Horpe. Sulla teoria dell' induzione unipolare. - L' esperienza descritta in un precedente lavoro, (cfr. $N$. Cim. vol. XXII) e che fu oseguita con un elettromagnete, $\dot{\theta}$ stata ripetuta con uguali resultati, adoprando in vece un magnete cilindrico cavo di acciaio. Oltre a ciò, non è riuscito all' A. di trovare una carica elettrostatica sulle basi del magnete ruotante, come dovrebbe resultare dalla teoria dell' induzione unipolare; perchè con un elettrometro a quadranti sensibilissimo ha sempre avuto resultati negativi. Cosi l'Autore ha concluso che nell'induzione unipolare devesi considerare solamente la parte fissa del circuito; cho la grandezza deli' inảuzione dipende solo dalla posizione dei due contatti striscianti e che è massima quando uno dei contatti $\dot{\theta}$ sull' asse di rotazione esternamente al magnete $\theta$ l'altro è nel mezzo fra' due poli e più prossimo che $\dot{e}$ possibile al magnete, Quando la distanza di questo secondo contatto dal magnete è sufficiente, i valori trovati concordano assai con quelli calcolati con la teoria del Wiedemann.

L'A. crede che sarebbe conveniente sostituire l'apparecchio da esso costruito a quello del Plucker per le dimostrazioni scolastiche sull' induzione unipolare, dacchè col suo apparato è elimincio ogrni dubbio sulla sede della forza elettromotrice.

In ultimo l' Hoppe replica alle osservazioni dell' Edlund ( $\nabla$. fasc. precedente) dich aracdo cbo egli con ha mai contestato la validita della formula usata da Edlund, che è la formula di Biot $\theta$ Savart; e che la divergenza f'ra esso e l'Edlund sta unicamente nel significato da attribuirsi alla quantita $v$ che comparisce in quella formula. Secondo Edlund $v \dot{\theta}$ una velocita assoluta; mentre secondo l' Hoppe l' induzione è possibile soltanto se il magnetismo e il rircuito indotto si spostano l' uno relativamente all' altro. Quanto alla svista che secondo Edlund sarebbe stata fatta dal. l'A., questi la nega, facendo vedere che si $\dot{\theta}$ attenuto esattamente a quanto è esposto nei lavori stessi di Edlund sull' origine dell'elettricità atmosferica.

Insiste poi nel ritenore cho durante la rotazione d' un magrete ruotino anche lo sue linee di forza, cid chè è regato da Edlund; ed osserva che tal rotazione potrebbe esser dimostrata sperimentalmente per mezzo dell' induzione esercitata su una sbarra di ferro disposta nella direzione dello linee di forza, circondando tale sbarra con un rocchetto incluso nel circuito di un elettromagnete. Nel vol. XXX, n. 4, di questi stessi Annali, l'Edlund replica anche a quoste osservazioni di Hoppe, e senza contare cho la teoria da questi proposta porterebbe a resultati contrari allo proposizioni fondamentali della tormodinamica, osserva che se I'Hoppe non ha ottenuto i resultati sperimentali che erano da 
aspettarsi, è stato perchò l' elettrometro adoperato non poteva essere abbastanza sensibile.

Quanto all' uso della formula di Biot, l' Edlund dice che dovè mostrarne l'applicabilità all' induzione unipolare, perchè fu stabilita per altro scopo. Insiste nel ritenere la validità della tcoria da esso proposta, e dice che le esperienze di Hoppe non fanno che confermarla sempre più.

F. HImstedT. Su una determinazione della quantità $v$. - Un condensatore la cui capacità è $\mathrm{Q}$ in misura elettrostatica, e $\mathrm{Q} / v^{2}$ in misura elettromagnetica si carichi e si scarichi $n$ volte al secondo col porre alternativamente lo sue armature in comunicazione cogli estremi di una resistenza $A B$, per mezzo della quale $\dot{\theta}$ chiusa una pila, e cogli estremi $D, D$, di una dolle spirali di un galvanometro differenziale. Le estremita F, F, dell' altra spirale del galvanometro sieno unite permanentemente con due punti $\mathrm{A}$ e C della resistenza $\mathrm{A} \mathrm{B}$, scelti in modo che il galvanometro non sia percorso da corrente; sia $W$ la resistenza fra $A$ e $C, R$ quella del circuito FF del galvanomotro, e W quella fra i punti A e B; allora si avrà

da cui

$$
n \mathrm{Q} / v^{2} \mathrm{~W} i=\frac{w i}{\mathrm{R}+w}
$$

$$
v=\sqrt{\frac{n \mathrm{QW}(\mathrm{R}+w)}{w}}
$$

Con questa formula, determinando in $m$ isura elettromagnetica il valore di $\mathrm{W}$ e del rapporto $(\mathrm{R}+w) / w, \mathrm{l}$ ' $\mathrm{A}$. ha trovato

$$
v=30,074 \cdot 10^{6} \frac{\mathrm{cm}}{\mathrm{sec}}
$$

Per misurare $n$ fu adoperato per interruttore un diapason elettromagnetico, che funzionava benissimo alla sola condizione cho il mercurio dei pozzetti nei quali venivano a pescare i filini di platino non avesse oscillazioni, e purchè $i$ due rebbi dol diapason abbiano la stessa massa.

R. Lampreht. Sull' azione del magnete sulle scariche elettriche nei gas rarefutti. - L' $\Lambda$. studia la forma che il filetto luminoso della scarica elettrica deve prendere, considerando un caso più generale di quello trattato in un suo precedente lavoro (vedi N. Cim. XIX, pag. 162). Supponendo che un conduttore senza peso, perfettamente flessibile, percorso da una corrente d'intensità J sia soggetto all' influenza di masse magnetiche distribuite comunque, e la cui funzione potenziale sia V, egli trova da prima 
86

che la tensione $T$ deve esser costante lungo tutto il conduttore, e che il raggio di curvatura della linea secondo cui si dispone

$$
p=\frac{\mathrm{T}}{\mathrm{AJ} \mathrm{P} \operatorname{sen}(\mathrm{P}, \overline{d s})},
$$

ove $\mathrm{P}$ è la forza che agisce sull' uniti di magnetismo sopposto concentrato nel punto $(x, y, z)$ in cui si considera la curvatura, $\theta$ $(\mathrm{P}, d s)$ è l'angolo che la direzione di quella forza fa con la direzione del filetto. A un resultato analogo conduce la legge di Biot o Savart; talchè si vede che il filetio luminoso non può disporsi secondo le linee di forza del campo altro che se tali linee sono rette; e ciò contradice a quanto è stato indicato da Hittorff.

A. Foeper. La distribuzicne della carica elettrica nei conduttori. - Ammettendo che il fluido elettrico sia elastico e libcramente mobile in ogni direzione, che agisca secondo la legge di Coulomb e che sia soggetto a forze elastiche che dipendano soltanto dalla densita elettrica nel punto considerato, (mentre il Maxwell ammetteva l' elasticità del fluido soltanto nei dielettrici), il Foeppl trova che l' elettricita libera si deve distribuiro alla superficie di un conduttore in uno strato sottilissimo. Ottiene poi una relazione fra le costanti fondamentali caratteristiche del fluido elettrico nel modo seguente. In un filo che è situato secondo l' asse delle X si propaghi un' onda elettrica longitudinale. Dalla teoria della elasticità si ha:

$$
\frac{d p}{d x}+\mu \frac{d^{2} \dot{\xi}}{d t^{2}}=0
$$

ove $\xi$ è lo spostamento di una particella nel senso dell' asse $X$, $\mu$ è la massa del fluido contenuto in $1^{\mathrm{cru}^{3}}, t$ il tempo e $p$ la pressione superficiale specifica, tale cioè che sia

$$
p=c . \Delta \varepsilon
$$

( $\Delta \varepsilon \dot{\theta}$ la pressione elastica dell' elettricità libera, che, per la estrema mobilita del fluido, si deve ritenere funzione solamente delle coordinate e indipendente dalla dirczione, e c è una costante clie ha le dimensioni di un potenziale).

Dalla rclazione precedente si ha:

$$
\frac{c}{\gamma} \frac{d^{2} \ddot{\xi}}{d x^{2}}=\frac{d}{d} \frac{\xi}{t^{3}},
$$

ove $\gamma$ è la massa dell' unità elettrostatica di elettricità. Dalla (2) si ha quindi

$$
c=w^{2} \gamma
$$


essendo $w$ la velocitá della propagazione della perturbazione elettrica.

Poichè $w$ è conosciuto, la (3) può servire a determinare una delle due quantità $c$ o $\gamma$, quando ne sia conosciuta una.

L. Bol.tzMann. Osservazioni sulla Memoria del sig. Lorberg su un soggetto di elettrodinamica. - Il Lorberg fece notare che nelle considerazioni che l'Aulinger feco sulle leggi dell' elettrodinamica, è contenuta una ipotesi che non è stata accennata esplicitamente nè da Hertz nà dall' Aulinger. Tale ipotesi consiste nell' ammettere che le forze elettriche cui è dovuta l' induzione sieno in grado di esercitare anche un' azione ponderomotrice. Il Boltzmann tratta teoricamente tale ipotesi, che potrebbe fornire un argomento decisivo per la teoria di Weber, quando fosse possibilo verificarla sperimentalmente.

W. VoIGT. Sulla torsione di un prisma rettangolo di sostanza cristallina omogenca. - Non è possibile accennare brevemente al modo con cui l'A. tratta analiticamente questo problema.

J. KIEwIET. Sulla elasticità di flessione dello zinco, dello stagno, del rame e delle loro leghe. - Dagli studi dell' A. resulta che l' elasticitá di una sostanza non è costante, e che per le leghe dipende dal loro stato, che puó esser diverso assai in vari casi, e che puó esser determinato dal modo con cui avviene la fusione dei componenti. Ordinariamente la variazione di E colla temperatura è esprimibile, fra 0 e $100^{n}$ c., per mezzo di una funzione lineare; ma da tale relazione per i netalli semplici, non si può dedurre quella relativa alle leghe che formano. Il peso specifico della lega, dedotto colla regola dei miscugli, $\dot{e}$ in generale esatto, specialmente se si determina con una porzione assai grande di sostanza. Per nessuno dei metalli studiati il rapporto \& fra la contrazione trasversale e quella longitudinale ha il valore $1 / 3 \mathrm{am}$ messo da Poisson.

J. Stefan. Relazione fra le teorie della capillarità e dell' evaporazione. - Alle considerazioni note che si fanno nella teoria della capillarità, l'A. ne aggiunge un' altra che si riferisce all' azione che il liquido esercita sulle particelle che sono fuori della sua massa, e la relazione indicata consiste nell' uguagliare il lavoro cho si richiede per portare una particella dalla sulerficio piana del liquido fino a una distanza superiore al raggio della sfera d'azione, con quello che è necessario per portare fino alla superficie una particella che si trovi fuori di quella sfera d'azione mclecolare.

A. Heritsch. Sulla radiofonia. - Una lastra assai spessa e lunga di casbone fu posta su una lampada Bunsen fino a portarla all' incandescenza, $\theta$ poi fu portata rapidamente in un tubo di ve- 
tro, prima che divenisse oscura, e sottoposta all' azione intermíttente dei raggi solari. I suoni prodotti così sembrano indebolirsi mano a mano che la lastra si raffredda, contrariamente all' ipotesi del Bell cho ammetteva che il suono fosse prodolto dal gas che era alternativamente espulso dai pori del carbone quando era soggetto all' azione dei raggi sulari, e poi riassorbito nel raffreddamento successivo. L' esperienza è stata ripetuta anche con la fiamma di una candela stearica, o di un 'nme a petrolio, su una lastra di carbone foggiata a lampada Ed:...i, e tenuta incandescente con una forte corrente elettrica; talchi l'A. conclude che nella radiofonia non entra per nulla l' assorbimento dei gas.

G. Konb. Sullo spettro del Germanio. - Sono indicate le lnnghezze d'onda di 16 lince dello spettro del Germanio.

Granier e Friedricils. Su una nuova pompa a mercurio. La novita consiste in un robinetto a due vie parallele e oblique, colle quali si può mettere l' estremità superiore della camera barometrica in comunicazione sia col tubo di aspirazione, sia con quello di espulsione.

A. Grosse. Reostato a nastro. - Un filo di argentana di mm. 0,15 di spessore è avvolto attorno un filo di cotono, il quale $\grave{e}$ poi tessuto in un nastro di $2 \mathrm{~cm}$. di larghezza, in modo che lo singole spiro del filo metallico siano fra loro isolate dalla sostanza coibente del tessuto. Una piccola porziono nel mezzo del nastro è scoperta per tutta la sua lunghezza, e un contatto metallico che scorre sulla porzione scoperta puó introdurre in circuito resistenze diverse. Con $4 \mathrm{~m}$. di nastro si giunge a una resistenza di 1000 ohm.

W. 'Hourz. Ponte di Wheatstone per l' efflusso dell' aria e delll' acqua. - Quattro tubi a T, di ottone, son riubiti fra loro in catena per mezzo di tubi di caucciù. Le aperture di due estremità opposte servono all' entrata e all' uscita del fluido; le altre due son riunite per mezzo di un largo tubo di vetro nel cui mezzo è un disco di cartone fissato lungo un diametro con due fili di seta, e girevole attorno ad essi.

Se i 4 tubi sono uguali fra loro, o se le resistenze delle duo parti dei due rami sono fra loro nello stesso rapporlo, nel tubo di vetro non si ha corrente, e il disco resta immobile. Un ponte analogo potrebbe costruirsi anche per l' efflusso del calore, con elementi termoelettrici.

Philosoph. Magazine - Vol. XXI, 1886.

132. - LORD RAYLEIGH. Reazioni in un sistema che eseguisce vibrazioni armoniche forzate, di periodi diversi. - Applicazioni all' elettricità. - Estendendo un teorema di Thomson sull' energia 
dei movimenti iniziali (Natural Phil. $\$ \S 316,317$ ) l' A. tratta alcuni casi speciali e ne fa l'applicazione alla distribuzione dell'elettricita, supponendo che le forzo agenti sul sistema, anzichè istantanee o costanti, siano funzioni periodiche del tempo. Egli trova molti importanti resultati; o partendo dalle equazioni generali del movimento, ottieno molto delle formule del Maxwell.

Detto. Sull' autoinduzione e sulla resistenza dei conduttori rettilinei. - Sono correzioni ed estensioni del metodo usato dal Maxwell nel suo noto Trextisc on Elcctricity.

A. P. LaUrie. Sulla misura della forza elettromotrice di una pila voltaica costante a elettrodi molili. - I' A. ritiene che la diminuzione che presenta la f. e. $m$. di una pila a circuito chiuso e che si attribuisce a polarizzazione, o a resistenza al passagr:o, sia dovuta in gran parte, e forse anche interamente, allo altorazioni negli strati del liquido che sono vicini agli elettrodi. Egli fece l' esperienza seguente. Una pila fatta con una lamina di platino di $90 \mathrm{~cm}$. q. e con una lamina di cadmio, aveva quest' ultima in moto per mezzo di un roteggio da orologio ( 2 o 3 rivoluzioni al secondo) La f. e. m. misurata col galvanometro, concorda con quella ottenuta coll' elettrometro: talchè si puó concludero che nella pila a jodo e cadmio, la f. e. m. misurata coll' elettrometro è quella dovuta alla formazione dell' ioduro di cadmio.

W. BaILy. Una carta del globo colla projezione di Flamsteed. - È noto che la proiezione di Flamsteed consiste nollo sviluppare in un piano $i$ paralleli della sfera celeste. L'A. ha usata questa proiezione per rappresentare la superficic del nostro globo, e ritione che sarebbe utile usarla in geografia, perchè i paralleli son cosi rappresentati da rette parallele equidistanti, e perchè $l^{\prime}$ area di una porzione qualunque è riprodotta senza alterazione.

C. Tominson. Nota sulle soluzioni saline soprasature. - L'A. ritiene col Nicol che una soluzione soprasatura sia una soluzione del sale anidro, e richiama alcune esperienze che fece in proposito fondandosi sulla diminuzione della forza elastica del vapore dovuta alla presenza di sali nell' acqua.

J. H. Poyntixg. Ia scarica elettrica in un isolante imperfetto. - La carica residua dei condensatori è spiegata dal Maxwell coll' ipotesi che il dielettrico sia un isolante imperfetto, la cui conducibilità varî da panto a punto. I'A. sostituisco a questa un' altra ipotesi, considerando $\mathrm{i}$ tubi d'indusione che in certo modo congiungono le due armature del condensatore. Se queste si scaricano mediante un filo metallico, e non si vuol toner conto del dielettrico interposto, si può ammettere che le cariche opposte si propaghino lungo il filo e si neutralizzino svolgendo calore; ma se si vuol tener conto del dielettrico, i tubi d'induzione si 
90

muoverebbero verso il filo, si accorcerebbero, mentre le loro estremita che rappresentano le cariche, si avvicinano indefinitamente e si annullano.

Questa è la parte essenziale della nuova ipotesi, che è dalI'A. sottoposta anche alle riprove del calcolo.

'T. E. THorps e A. W. Rücker. Nota su una relazione fra le temperature critiche dei corpi e $i$ loro coefficienti di dilatazione allo stcto liquido. - A proposito di una Nota dei prof. Bartoli e Stracciati ( $N$. Cim. XVI, pag. 91) gli A. fanno osservare che la critica fatta alla loro formula mostra solamente che quella formula non $\dot{\theta}$ valida in certe circostanze, nelle quali gli $A$. stessi non hanno mai inteso di adoperarla, perchè è solamente una formula di approssimazione. Insistono poi sul fatto che, come legge di approssimazione, la formula di Mendelejeff

$$
\mathrm{V}_{\mathrm{t}}=\frac{1}{1-\mathrm{K} t}
$$

è quella che, meglio assai delle altre

$$
V_{\imath}=1+a t+b t^{2}+c t^{3}+\ldots \ldots
$$

rappresenta i resultati sperimentali fra $0^{0}$ e il punto di ebollizione doi liquidi. I proff. Bartoli e Stracciati hanno peraltro replicato (Ph. Mag. 1886, $\mathrm{n}^{0}$ 139) che non ebbero intenzione di criticare il lavoro dei sigg. Thorpe e Rücker, ma di mostrare soltanto, con numerosi esempi, i limiti entro $i$ quali le formule di Mendelejeff e di Thorpe e Rücker sono applicabili.

Balfour StewarT. Sulla causa delle variazioni del magnetismo terrestre riferite al giorno solare. - L'A. discute le ipotesi fatte fin qui in proposito, $\theta$ crede che nessuna sia sufficiente a spiegare $\mathrm{i}$ fenomeni osservati; dice che la causa delle variazioni magnetiche diurne $\dot{e}$ da cercarsi al di fuori della 'Terra; ma non nel Sole, bensi nelle parti superiori dell' atmosfera.

133. - Lord RAYLeigri. Note, principalmente storiche, su alcune proposizioni fondamentali dell' ottica. - Son richiamati alcuni teoremi importanti stabiliti da Cotes e da Smith (e che poi furon ritrovati da Lagrange, da Kirchhoff o da Helmholtz) riguardanti principalmente la distanza apparente alla quale vien veduto un oggetto attraverso un numero qualunque di lenti, all' ingrandimento degli strumenti ottici, e all' intensità luminosa delle inmagini.

S. P. Thompson. Su alcuni nuovi prismi polarizzatori. - Vien descritto un prisma costruito da Ahrens, formato con 3 prismi di spato calcare, che riuniti formano un parallelepipedo retto, una 
delle cui basi è quasi quadrata. Sugli altri prismi esso ha il vantaggio di esser piú corto, di aver maggior apertura angolare, di rifletter minor quantita di luce perchè non ha facce oblique, di aver poca distorsione $\theta$ di richiedere minor quantita di spato in confronto degli altri prismi di ugual sezione (Hartnack, Glan, Thompson ) eccetto $i$ nicol.

Il prisma di Nicol può esser modificato vantaggiosamente, col tagliare le due parti in modo che il piano di unione faccia un angolo quasi retto con l'asse cristallografico e colla faccia terminale. Cosi si puó costruire il prisma con meno spato, e non si ha l'iridescenza bleu dei soliti nicol.

S. H. BdRBdry. I fondamenti della teoria cinetica dei gas. Nota sulla Memoria del prof. Tait. - L' A. mostra che una dello ipotesi fatte dal Tait può esser tralasciata.

C. Tomlinson. Osservazioni su una nuova teoria d:lla rugiada. - Sono osservazioni critiche e storiche sulla teoria proposta dal sig. Aitken, dalle quali l'A. conclude che non $\dot{\theta}$ possibile ammettere che la rugiada sia dovuta ai vapori che durante la notte vengano emessi dal suolo; ma che invece essa è dovuta alla condensazione del vapor acqueo contenuto nell' atmosfera, come fu stabilito dal dott. Wells.

A questo l' Aitken ha replicato ( $P h . M a g .1886$, n. 135 è 137) facendo notare che egli non ha inteso di dare una nuova teoria, in opposizione a quella del Wells, bensi di estendere il lavoro i cui fondamenti furon posti dal Wells. Il Tomlinson peraltro (Id. n. 136) dice che anche nei giornali che l'hanno riprodotta, la teoria dell' Aitken è indicata come nuova e opposta a quella del Wells. Sembra peraltro che gli A. non abbiano tenuto conto degli studi del Fusinieri, il quale, nelle sue controversie col Wells a col Melloni, osservò e discusse alcuni dei fatti che sono stati posti dall' Aitken a base del suo lavoro.

H. WILDE. Sull' effusso dell' aria modificato dalla forma dell' orifizio di scarica. - In seguito alle osservazioni del prof. 0. Reynolds (cfr. N. Cim. XXII, p. 92) l' A. ha fatto altre osperienze con orifizi di varia forma praticati in lastre di vario spessore $\theta$ ha trovato che il valore del coefficiente di contrazione, che $\dot{o}$ stato applicato per l' efflusso dell' aria ad una pressione inferiore di 15 libbre per pollice quadrato, $\dot{\theta}$ quasi del tutto dovuto al cambiamento di resistenza dal recipiente che contiene il gas a quello che lo riceve, $\theta$ non dalla forma dell' orifizio nè dalla lunghezza delle aggiunte, come avviene pei fluidi non elastici. Da queste nhove esperienze il valore di tal coefficente resulterebbe 0,937 ; e con questo valore la velocità con la quale l'aria atmosferica alla pressione assoluta di 15 libbre si scarica nel vuoto, attraverso un ori- 
fizio della forma più opportuna, sarebbe di 677 piedi al secondo; e per gli altri gas è facile ottenere la velocita corrispondente servendosi della nota legge di Graham.

J. Y. EDGEivortif. Sulla determinazione del modulo degli errori. - Sono considerazioni intese a facilitare $i$ calcoli che son necessari ad ottenere il valore di quel modulo.

134. - O. Fisher. Sulle variazioni della gravitì in alcune stazioni dell' arco di meridiano indiano, e loro relazione colla costituzime della crosta terrestre. - Discutendo le osservazioni fatte col pendolo in diversi luoghi dell'India, riferite dal general Walker alla riunione della Associazione Britannica in Aberdeen nel 1885, l'A. conclude che la teoria dell' equilibrio idrostatico esposta nella sua "Physics of the Earth's Crust, ne $\dot{\theta}$ confermata, e che quindi $\dot{\theta}$ anche ammissibile l'ipotesi fatta che la densita media della crosta terrestre sia 2,68.

S. Bidwell. Modificazione del reostato di Wheatstone. - II filo $\dot{e}$ avvolto su un cilindro di legno, ed è fissato ai due capi. Il cilindro è montato come quello di Duhamel, e un contatto fisso striscia sul filo e introduce nel circuito più o meno filo a seconda della posizione che occupa il cilindro di fronte al contatto anzidetto.

W. Ravsay e S. Younc. Alcune relazioni termodinamiche. Parti IV. e V. - Gli A. replicano alle osservazioni fatte ai loro precedenti lavori dai proff. Ayrton e Perry (cfr. N. Cimento) dicendo che non intesero dare relazioni teoriche, ma molto approssimate; e riconoscono che avrebbero risparmiato molti calcoli se avessero notata l'identita delle diverse relazioni da essi studiate.

W. Ostwald. Sulla sede della f. e. m. nelle pile voltaiche. L'A. accetia le conclusioni del prof. Lodge, e indica un metodo per ottenere direttamente la differenza di potenziale fra due liquidi, o fra un liquido e un metallo. Il metodo si basa sull'osservazione di Helmboltz che se un filetto di mercurio cade, da un recipiente isolato, sulla superficie di un liquido, acquista rapidamente il potenziale del liquido. Così se una coppia di quadranti dell' elettrometro è in comunicazione col mercurio che scola nell'interno di un liquido $F$ e se l'altra coppia è in comunicazione con una lastra metallica M che pesca nel liquido, l'elettrometro indica la differenza di potenziale fra $F$ ed $\mathbf{M}$. Nel caso di due liquidi $F_{1}$ e $F_{2}$, si pongono in recipienti separati, che comunicano con un terzo per mezzo di piccoli sifoni. Due filetti di mercuris che cadono nei due liquidi respettivamente, e che sono in comunicazione colle due coppie di quadranti dell' elettrometro, completano l'apparecchio necessario per trovare la differenza di potenziale fra $i$ liquidi $F_{1} \in F_{2}$. 
135. - W. StTHerlaxo. Ia legge di altrazione fra le molecole di un gas. - In questo lungo lavoro I'A. dimostra che le esperienze di Thomson e Joule conducono alla legge che le molecole di un gas agiscano fra loro in ragione composta delle loro masse $e$ in ragione inversa della $4^{a}$ poteuza delle distanze.

R. SIImA. Nuovo strumento per registrare continuamente la intensità e la direzione di una corrente elettrica variabile. - È la descrizione di un apparecchio che si puo sostituire a quelli soliti fondati sulla fotografia, e che puo lavorare anche in ambienti illuminati. L'A. I' ha adoprato per registrare antomaticamente lo correnti telluriche. Il prineipio dell' apparecchio è quello stesso del Siphon Recorder del Thomson; ma non è possibile descriverlo senza l' aiuto di disegni.

W. OstwaldD. Ricerche elettrochimiche. - Secondo la teoria di Clansius dell' elettrolisi e quella del Williamson delle reazioni chimiche, deve esistere un' intima connessione fra le reazioui chimiche degli acidi $\theta$ la loro resistenza elettrica. Secondo Williamson infatti la velocità delle reazioni chimiche deve dipendere dalla velociti con cui gli atomi si scambiano fra loro; o la teoria di Clausius dice che la conduttivita elettrolitica si deve al fatto cho gli ioni spostano continuamente clementi o radicali equivalenti dalle molecole, e che le parti delle molecolo che son poste in liburtà si scambiano con altro simili. Quanto più rapidamente gli ioni potranno scambiarsi e tanto meglio la elettricità potrà passare pel liquido. Ora poichè secondo Faraday, gli ioni trasmetton sempre la stessa quantità di elettricità, indipendentemente dalla loro natura chimica, dallo duo teorio anzi dette bisogna concludere che le velocità dei cambiamenti chimici prodotti dagli acidi devono esser proporzionali alla velocita con la quale essi trasmettono quantità uguali di elettricità ciò̀ alla loro conduttività elettrica.

Questa conclusione, che $\mathrm{fu}$ indicata nel 1881 dall'Arrenius e da esso verificata per un piccol numero di casi, o stata riconosciuta esatta dall' Ostwald per alcune reazioni prodotte da un gran numero di aoidi, pei quali egli aveva già determinato la velocitd delle reazioni stesse.

Per rappresentare i resultati ottenuti l'A. non ha considerato Ia conduttivita specifica degli acidi, ma in vece la conduttivitz molecolare, che egli definisce cosi: Se in un recipiente che contiene due elettrodi paralleli, distanti $1 \mathrm{~cm}$, si pone un peso in grammi uguale al peso molecolare dell' acido, la conduttività dell'acido, quando è diluito con una quantità definita di acqua, è la conduttività molecolare per quol grado di diluizione. Questo grado di diluizione poi è espresso dal numcro di litri di soluzione che 
94

contiene in grammi un peso del dato acido uguale al suo peso molecolare.

Se la diluizione è zcro, ciò̀ se non vi è acqua, la conduttività di un acido è ordinariamente nulla o poco diversa da zero. Aggiungendo acqua la conduttivita cresce, e tende a un massimo che è raggiunto quando la diluizione è infinita; e l' A. osserva che non vi è eccezione a questa legge generale. Le conduttivita degli acidi più energici, come $\mathrm{HCl}, \mathrm{HBr}, \mathrm{HJ}, \mathrm{HNO}_{3}, \mathrm{HClO}_{3}$, ecc., hanno quasi il loro massimo in diluizioni moderate, e la conduttività molecolare di quegli acidi varia poco con la diluizione; il loro massimo, quasi uguale per tutti, è raggiunto a una diluizione di circa 512 litri; in soluzioni più diluite la conduttivita diminuisce accennando alle impurità contenute nell' acqua distillata.

I resultati ottenuti per gli acidi monobasici posson esser rappresentati con la formula empirica

$$
+\operatorname{tang} m=\left(\frac{v}{v_{0}}\right)^{0.4124}
$$

ove $m$ ò la conduttività molecolare espressa in funzione di quella massima posta uguale a $90, v$ è la quantita di acqua che esprimo il grado di diluizione, $\Theta v_{0} \dot{\theta}$ una costante dipendente dalla natura dell' acido.

Gli acidi polibasici hanno un contegno piủ complicato, e per essi l'A. non ha potuto trovare un' espressione analitica che rappresenti bene $\mathrm{i}$ resultati ottenuti.

O. Heaviside. Sull' autcind uzione nei fili. - A proposito delle esperienze note del prof. Hughes, l'A. espone i resultati cui è giunto trattando analiticamente il problema della propagazione della elettricità nei fili; ma delle lunghe Note pubblicate in diversi fascicoli del Phil. Mag. non è possibile dare un breve sunto.

H. Cunynghame. Nuovo iporbolografo. - Su una riga di legno scorre una doppia squadra, e sul pezzo di questa che è perpendicolare alla riga, scorre un altro pezzo di legno, che porta una matita, al quale è fissato uno dei capi di un filo di cui l'altro capo è fissato a un corsoio mobile lungo una guida normale alla riga. Il filo passa su una puleggia $\operatorname{che}$ è portata dalla doppia squadra nel punto in cui essa si appoggia alla riga; ed è chiaro che facendo scorrere la doppia squadra sulla riga, la matita descrive un' iperbola.

A. B. BAsset. Nota sull' induzione di correnti elettriche in una sottil lastra piana indefinita, che ruota in un campo magnetico. Applicando il metodo dello immagini, L' A. ottiene le stesse formule già trovate dal Niven coll' uso delle equazioni della forzb elettromotrice riferita ad assi mobili. 
W. Emmotт e W. Ackroyd. Su un indicatore a luce elettrica del grisou. - Di due lampade a incandescenza fissate su uno stesso sostegno una ha il votro bianco, l'altra lo ha verde. In condizioni normali è accesa la lampada bianca; ma per mezzo di un vaso poroso e di un indice mobile di mercurio o di acido solforico, viene introdotta nel circuito della pila la lampada verde, quando il metano comincia a mescolarsi coll' aria.

S. P. LaxoLfy. Sulle lunghezze d' onda fino ad ora non misurate. - Sono descritti gli apparecchi che l'A. ha usato nelle sue ricerche, $\theta$ sono esposti i resultati da esse ottenuti: risultati dei quali furon gia dati dei cenni in questo Giornale $(X V$, p. 184 e XX pag. 43).

A. Buchumin. Estensione di un teorema del prof. Silvester, circa le matrici. - L'A. applica al caso di radici uguali un teorema d'interpolazione che il Silvester dimostrò per le radici disuguali.

Lord. RAPLEIGi. Note sul magnetismo - I Energia del ferro magnetizzato. - Dopo l' esempio di Rowland e Stoletow, i resultati delle esperienze sui magneti si rappresentano con delle curve che indichino la relazione fra l' induzione magnetica $B$ e la forza magnetizzante $\mathrm{H}$. In un ciclo di operazioni, durante if quale $\mathrm{H}$ dapprima aumenta e poi diminuisce, l'induzione $\mathrm{B}$ \& sempre maggiore nella parte discendente che in quella ascendente della curva, e il Warburg ha mostrato che il lavoro speso nel far percorrere al ferro un ciclo magnetico $\dot{\theta}$ rappresentato $d a-\int \mathrm{I} d \mathrm{H}$, ove $\mathrm{I}$ $\dot{\theta}$ il magnetismo libero; ovvero, per un ciclo chiuso, da $1 / 6 \pi$ $\int \mathrm{B} d \mathrm{H}$, perchè, essendo sempre $\mathrm{B}=\mathrm{H}+4 \pi \mathrm{I}$, in un ciclo chiuso $\int \mathrm{H} d \mathrm{H}$ è nullo. In questo caso si ha anche

$$
-\int \mathrm{I} d \mathrm{H}=\int \mathrm{H} d \mathrm{I}=1 / 4 \pi \int \mathrm{H} d \mathrm{~B} \text {. }
$$

Ma nei cicli chiusi è più utile prender I per variabile indipendente, come per es. nella magnetizzazione del ferro dolce colle correnti. Allora in un anello di ferro di lunghezza $l$ e di sezione $\sigma$, con $n$ spire percorse da una corrente $C$, la f. $e . \mathrm{m}$. totale inducente è $n \sigma d \mathrm{~B}$, e l' elemento di lavoro $\dot{\theta} n \sigma \mathrm{CdB}$. E poichè $l \mathrm{H}$ $=4 \pi n \mathrm{C}$, l' elemento di laroro per l'unità di volume del ferro è

$$
1 / 4 \pi \mathrm{H} d \mathrm{~B}=1 / 4 \pi \mathrm{H} d \mathrm{H}+\mathrm{H} d \mathrm{I} \text {, }
$$

ove l' ultimo termine è dovuto specialmente al ferro. In pratica 1/. $\pi \mathrm{H} d \mathrm{H}$ è trascurabile, e allora si può prendere $1 / 4 \pi \mathrm{H} d \mathrm{~B}$ in luogo 
96

di $\mathrm{H} d \mathrm{I}$. Ma non si posson mai scambiar fra loro $\mathrm{BdH}$ \& $\mathrm{H} d \mathrm{~B}$; per il ferro che sia già stato magnetizzato quasi a saturazione, $\theta$ che si sottoponga a una forza magnetizzante crescente, si ha sempre

$$
\int \mathrm{B} d \mathrm{H}>\int \mathrm{H} d \mathrm{~B}
$$

E un fatto che da una corta sbarra di ferro magnetizzata si può riottenere dell' energia; ma questa dipende dalla polarità libera alle estremita. Il lavoro immagazzinato ò quello che potrebbe ottenersi se, la sbarra essendo supposta flessibile, si facessero avvicinare l' una all' altra le sue estremità, per la loro mutua attrazione. Finita quest' operazione, quando ció la sbarra sia divenuta un anello, non si può piu ottenere da essa nessun lavoro, quantunque resti magnetizzato. Perciò l' A. contro il parere dell' Ewing, riteneva che un circuito magnetico chiuso sia da evitarsi nei generatori secondari o nei rocchetti $d$ 'induzione, poichè in esso la massima parte dell' energia comunicatagli è perduta. Lo stesso vale per l' uso di elettromagneti chiusi posti in circuiti percorsi da correnti alternate. Ma in una nota successiva l'A. dice che l'Ewing e il D.' Hopkinson gli focero osservare che il rendimento dei rocchetti d' induzione non dipende dall' accumulamento dell' energia, $\theta$ che quei rocchetti posson lavorare con grande rendimonto anche se tutta l'energia comunicata al forro andasse perduta.

Calcolando poi il momento magnetico di un ellissoide di ferro, l'A. trova che il massimo si ha quando l' ellissoide é molto schiacciato. Nel caso delle lastre piane magnetizzate perpendicolarmente alla loro superficie, quasi tutta l'onergia spesa è riottenibile; ma in pratica poco si prestano tali lastre, perchè non si posson magnetizzare abbastanza fortemente.

La resistenza di un filo di ferro $\theta$ poi maggiore per le correnti variabili che per quelle continue, anche se la corrente è costretta a distribuirsi uniformemente nella sezione del filo. Mancando questa distribuzione uniforme, la resistenza cresce ancor più per la tendenza ad accumularsi sulla superficie esterna.

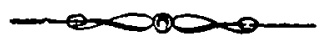

